\title{
Research on the Influence Mechanism of Meaning in Life on Subjective Well-being of Military College Students
}

\author{
Tian Yanhui ${ }^{1, a^{*}}$, Wang Haishan ${ }^{1, b}$, Bai Shengjun ${ }^{1, c}$, Wei Ting ${ }^{1, d}$ \\ ${ }^{1}$ college of Information and Communication, National University of Defense Technology, Xi'an, Shaanxi, China \\ a*tianyanhui05@163.com \\ b278870421@qq.com \\ cwmhuihd@163.com \\ dweiting06@163.com
}

\begin{abstract}
The purpose of this study was to explore the influence factors and mechanism of subjective well-being among military college students, and providing effective evidence for psychological intervention. A total of 413 military college students were investigated with meaning in life questionnaire, mindful attention awareness scale and index of well-being scale, the results shows that the correlation between meaning in life and mindful attention awareness was positive significant $(\mathrm{r}=0.18, \mathrm{P}<0.05)$, and also the correlation between meaning in life and index of well-being was positive significant $(\mathrm{r}=0.26, \mathrm{P}<0.01)$, finally, the correlation between mindful attention awareness and index of well-being was also reached a significant level $(\mathrm{r}=0.23, \mathrm{P}<0.01)$. The results of hierarchical regression analysis shows that, the meaning in life had a significant positive effect on the subjective well-being of military college students $(\beta=0.22, \mathrm{p}<0.001)$, and the mindful attention awareness played a partially mediation role between the meaning in life and subjective well-being, and it's size was 0.03 . The conclusion also has given some enlightenment significance for education and management of military college students, which we could improve the meaning in life and mindfulness awareness of military college students, and finally then improved their subjective well-being level.
\end{abstract}

Keywords: Meaning in life, mindful attention awareness, index of well-being, military college student

\section{军校大学生生命意义感对主观幸福感的影响机制研究}

田艳辉 $1, \mathrm{a}^{*}$ 王海山 ${ }^{1, \mathrm{~b}}$ 白胜军 $1, \mathrm{c}$ 魏婷 $1, \mathrm{~d}$

\author{
${ }^{1}$ 国防科技大学信息通信学院, 西安, 陕西, 中国 \\ a*tianyanhui05@163.com \\ b278870421@qq.com \\ cwmhuihd@163.com \\ dweiting06@163.com
}

\begin{abstract}
摘要: 探讨军校大学生主观幸福感的影响因素及作用机制, 为其进行有效心理干预提供依据。采用生命意义感 量表、正念注意觉知量表和主观幸福感量表对 413 名在校军校大学生进行调查, 结果显示, 军校学员生命意义 感与正念注意觉知 $(r=0.18, \mathrm{P}<0.05)$ 、主观幸福感 $(r=0.26, \mathrm{P}<0.01)$ 显著正相关，正念注意觉知与主观幸福 感间（r=0.23, $\mathrm{P}<0.01)$ 的正相关也达到显著性水平。层次回归分析的结果显示，军校大学生生命意义感对主 观幸福感具有显著的正向影响作用 $(\beta=0.22, P<0.001)$, 正念注意觉知在生命意义感和主观幸福感起部分中介 作用, 效应值为 0.03 。该结论对军校大学生的日常教育和管理也具有一定的启示意义, 可以从提高军校大学生 的生命意义感和正念注意觉知两个方面入手，进而提高其主观幸福感水平。
\end{abstract}


关键词: 生命意义感, 正念, 主观幸福感, 军校大学生

\section{1. 引言}

军校大学生是军队未来建设和发展的关键, 更是 部队战斗力生成的重要人力资源支撑。然而, 对于多 数军校大学生来说, 刚刚步入军营, 尚未脱掉 “孩子 气”, 就要练就 “英雄胆”, 稚气未脱的军校大学生在 面对生活学习环境改变和高强度军事训练时, 难免会 产生水土不服, 进而引发心理危机。近些年来, 军校 大学生出现心理预警数量呈明显上升趋势, 严重心理 疾患数量也有所增加, 与同龄地方大学生相比, 军校 学员所面临的压力更大, 更容易出现诸如焦虑、抑郁、 情绪表达等心理问题 ${ }^{[1]}$ 。因此, 关注军校大学生的心 理健康, 探讨其保护性因素和作用机制, 无论是对军 校大学生个人的成长进步, 还是对部队的安全稳定乃 至战斗力的提升都将起到重要的意义和价值。

在众多心理健康的指标体系中, 主观幸福感近些 年来收到了学术界的普遍关注, “你幸福吗？”甚至成 为了年度的流行词, 这也充分反映了在物质文明充分 发展后人们对生活品质所提出的新要求。主观幸福感 指的是个体依据自身标准对当前生活质量的总体评 价, 包括情感体验（正性情感和负性情感）和认知评 价 (总体生活满意度) 两个部分 ${ }^{[2]}$, 主观幸福感是积 极心理学的重要研究内容之一, 也是衡量个体心理健 康水平的核心指标, 有研究表明, 高主观幸福感的个 体生理上更健康更长寿, 事业上更成功 ${ }^{[3]}$ 。基于此, 本研究将主观幸福感作为军校大学生心理健康状况 的指标, 探讨其影响因素及内部作用机制, 以期为军 校大学生的管理和心理干预提供必要的理论依据。生 命意义感指的是个体感觉到自己的生活可以被理解, 自己被有价值的目标指引并且感到自己的生活有价 值时的感受 ${ }^{[4]}$, 研究表明, 生命意义感与快乐、生活 满意度、身体健康等一系列积极指标显著正相关, 而 与焦虑、抑有、PTSD、酒精和毒品滥用等消极指标显 著负相关 ${ }^{[5]}$ 。关于生命意义感与主观幸福感关系的研 究中, 牛端的研究表明, 生命意义感对科技人员的主 观幸福感具有显著的正相关关系 ${ }^{[6]}$; 谢其利等人则以 老年人群体为对象, 验证了生命意义感影响主观幸福 感的跨群体适用性。生命意义感描述了个体感觉到自 己的人生有意义、有目标、有价值的程度, 可以帮助 人们确立人生目标, 提升自我价值 ${ }^{[7]}$, 在生命意义感 的驱动下, 个体的行为会更具使命感, 行为的持久性 和动力性也更强, 即使面对眼前的困境, 也往往能够 调整看待问题的视角, 赋予困境以积极的意义, 进而 拥有积极的归因方式和良好的自我效能感。因此, 本 研究认为, 军校大学生生命意义感会对其主观幸福感
产生积极的促进作用。

生命意义感作为一种底层的、基础性的生活态度, 其对主观幸福感的影响可能并不是直接的, 而是通过 某种行为方式实现, 正念可能在该过程中扮演中介作 用。正念指的是个体有目的地将注意力维持在当前的 体验或某种刺激上, 而不做任何判断和评价的心理特 质和行为方式 ${ }^{[8]}$, 是一种起源于禅学的心理治疗方法, 正念疗法能够促进个体更好地适应环境, 维持个体的 情绪稳定。多数研究表明, 正念干预能够有效降低个 体的焦虑、抑郁等负性情绪, 减少反社会性行为, 同 时提高幸福感 $\left.{ }^{[9,} 10\right]$ 。生命意义感和正念关系的研究目 前少有涉及, 王秦飞和黄灵等人虽然证实了生命意义 感和正念间的正相关关系, 但在验证其内在因果关系 时普遍认为正念是生命意义感的原因而非结果 ${ }^{[11,}{ }^{12]}$ 。 弗兰克认为, 人类最基本的动机就是追寻生命的意义, 当一个人缺乏生命意义感时, 就会产生无聊、空虚、 厌烦等感觉, 严重者甚至产生神经官能症 ${ }^{[13]}$, 而当一 个人充满生命意义感时, 就会赋予周围的事物和情境 以特定意义, 用更加积极的态度对待周围的人和工作, 而不会将其视为累赘, 对待生活也更为专注, 用心做 好每一件事。基于以上分析, 本研究认为, 正念可能 是生命意义感的结果而非原因, 并据此构建了以正念 为中介变量的研究模型, 如图 1 所示。

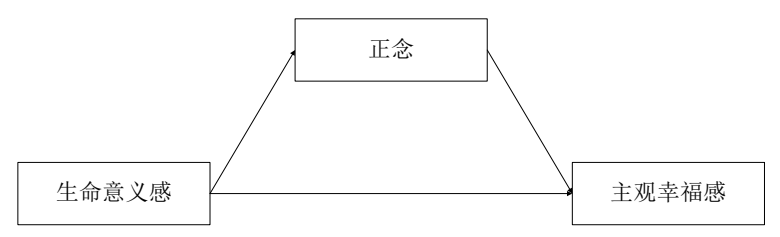

图 1 研究模型图

\section{2. 研究方法}

\section{1. 研究对象}

采用整群方便抽样的方法, 从西安、武汉、南京、 长沙等四个城市的四所军校中抽取 10 个教学班共计 413 名大学生作为本研究的调查对象, 共发放调查问 卷 413 份, 收回 398 份, 剔出无效问卷, 共得到有效 调查问卷 376 份, 有效收回率为 $94.5 \%$ 。调查对象的 人口学变量分布如下: 男生 293 人 $(77.9 \%)$, 女生 83 人 $(22.1 \%)$; 大一学生 241 人 (64.1\%), 大二学生 87 人 (23. $1 \%)$, 大三学生 48 人 (12.8\%); 政治面貌中, 党员 31 人 $(8.2 \%)$, 团员 335 人 $(89.1 \%)$, 群众 10 人 (2.7\%); 入学前身份分布中, 士兵为 193 人(51.3\%), 
士官为 124 人 $(33.0 \%)$ ，地方青年为 59 人 (15.7\%); 调查对象的年龄分布中,最小的 19 岁,最大的 27 岁, 均值为 21.7 岁, 标准差为 1.30 岁。

\section{2. 研究工具}

\subsection{1. 生命意义感量表 (the Meaning in Life Questionnaire, MLQ)}

采用 Stege 编写 ${ }^{[14]}$, 刘思斯等人修订的生命意义 感量表 ${ }^{[15]}$, 原始量表共包括 10 个项目, 修订后的量 表共包括 9 个项目, 归属于生命意义感和寻求意义感 两个维度, 采用 Likert7 点计分, 1-7 分别代表 “完 全不符合-完全符合” ，得分越高表明生命意义感越 强。本研究中, 两个维度的 Cronbach' $s$ a 系数分 别为 0.703 和 0.778 , 总量表的 Cronbach' $\mathrm{s}$ a 系 数为 0.843 。

\subsection{2. 正念注意觉知量表 (Mindful Attention Awareness Scale, MAAS)}

采用 Brown 编写的 15 题项正念注意觉知量表 ${ }^{[16]}$, 该量表也是目前测量正念水平时最为常用的评估工 具, 采用 Likert5 点计分, 1-5 分别代表 “几乎总是 一几乎没有”，所有题项均采用反向描述，得分越高正 念知觉越强。本量表为单维结构, 分别从个体的认知、 情绪和生理等方面进行总体评估, 本研究中其 Cronbach’ $\mathrm{s}$ a 系数为 0.944 。

\section{2. 3. 主观幸福感量表(Index of Well-Being; IWB )}

采用 Campbe11 编写的幸福感指数量表 ${ }^{[17]}$, 该量 表包含总体情感指数量表和生活满意度问卷两部分, 前者由 8 个项目组成, 分别从不同角度描述情感的内 涵, 后者只有 1 项。计算总分时将总体情感指数量表 的平均得分与生活满意度问卷的得分（权重为 1.1） 相加, 得分越高, 感知到的幸福感越强。本研究中,
其 Cronbach’ $\mathrm{s}$ a 系数为 0.949 。

\section{3. 数据处理}

将收集到的数据录入 SPSS23. 0 , 采用信度分析的 方法验证研究工具的可靠性, 采用皮尔逊积差相关检 验变量间的相关关系, 为后续因果关系的检验提供基 础, 采用层次回归分析的方法检验生命意义感对主观 幸福感的影响及正念在该过程中的中介作用。使用的 统计软件为 SPSS 23. 0 和 Hayes 基于 SPSS/SAS 开发的 PROCESS 宏程序 3.3。

\section{3. 研究结果}

\section{1. 共同方法偏差检验}

由于本研究中所使用的问卷均为调查对象自评, 不可避免受到共同方法偏差的影响, 本研究采用 Harman 单因素法对可能存在的方法学效应进行了检 验。将调查涉及到的所有题型进行探索性因素分析, 未旋转因子分析的结果显示, 共可析出 5 个特征根大 于 1 的公因子, 其中第一公因子特征根为 10.22 , 可 解释总方差变异 $30.97 \%$, 低于 $40 \%$ 的经验标准。因此, 可认为本研究中不存在严重的共同方法偏差。

\section{2. 变量间的相关分析}

变量的均值、标准差及彼此间的相关关系如表 1 所示。人口学变量与主要研究变量间的相关关系中, 年级与生命意义感 $(r=-0.21, p<0.01)$ 、正念 $(r=-0.13$, $p<0.01)$ 、主观幸福感 $(r=-0.23, p<0.01)$ 显著负相 关; 入学前身份与生命意义感 $(r=-0.13, p<0.05)$ 、 正念 $(r=-0.13, p<0.05)$ 、主观幸福感 $(r=-0.13$, $\mathrm{p}<0.05$ ) 显著负相关。主要研究变量间均呈现显著的 正相关关系，其中，生命意义感与正念（ $r=0.18$, $p<0.05)$ 和主观幸福感 $(r=0.26, p<0.01)$ 显著正相 关; 正念与主观幸福感显著正相关 $(r=0.23, p<0.01)$ 。 以上变量间的相关为后续假设检验提供了基础。

表 1 变量的均值、标准差及相关关系

\begin{tabular}{|l|c|c|c|c|c|c|c|c|c|}
\hline 变量 & $\mathrm{M} \pm \mathrm{SD}$ & 1 & 2 & 3 & 4 & 5 & 6 & 7 & 8 \\
\hline 1 性别 & $1.22 \pm 0.42$ & 1 & & & & & & & \\
\hline 2 年龄 & $21.70 \pm 1.30$ & $0.17^{* *}$ & 1 & & & & & & \\
\hline 3 年级 & $1.49 \pm 0.71$ & $-0.12^{*}$ & 0.03 & 1 & & & & & \\
\hline 4 政治面貌 & $1.94 \pm 0.33$ & 0.01 & 0.01 & $-0.31^{* *}$ & 1 & & & & \\
\hline 5 入学前身份 & $1.64 \pm 0.74$ & $-0.11^{*}$ & 0.10 & $0.58^{* *}$ & $-0.32^{* *}$ & 1 & & & \\
\hline 6 生命意义感 & $5.32 \pm 1.09$ & 0.06 & -0.04 & $-0.21^{* *}$ & 0.01 & $-0.13^{*}$ & 1 & & \\
\hline 7 正念 & $4.55 \pm 1.06$ & 0.08 & 0.03 & $-0.13^{* *}$ & -0.09 & $-0.13^{*}$ & $0.18^{*}$ & 1 & \\
\hline 8 主观幸福感 & $11.15 \pm 3.01$ & -0.08 & -0.04 & $-0.23^{* *}$ & 0.09 & $-0.13^{*}$ & $0.26^{* *}$ & $0.23^{* *}$ & 1 \\
\hline
\end{tabular}

注: ${ }^{*}$ 表示 $p<0.05,{ }^{* *}$ 表示 $p<0.01$. 


\section{3 正念在生命意义感和主观幸福感间的中介 作用}

根据温忠麟 ${ }^{[18]}$ 等检验中介效应的程序对正念在 生命意义感和主观幸福感间的中介作用进行检验，结 果如表 2 所示。第一步, 做主观幸福感对的生命意义 感回归, 结果显示生命意义感对主观幸福感具有显著 的正向影响 $(\beta=0.22, p<0.001)$, 如 M1 所示; 第二 步, 做正念对生命意义感的回归, 结果显示生命意义 感对正念的预测作用同样显著（ $\beta=0.14, \mathrm{p}<0.001)$,
如 M3 所示; 第三步，同时做主观幸福感对生命意义 感和正念的回归, 结果显示, 生命意义感对主观幸福 感具有显著的正向预测作用 $(\beta=0.19, p<0.001)$, 正 念对主观幸福感的作用也到显著性水平 ( $\beta$ $=0.20, p<0.001$ ), 如 M4 所示。由此说明, 正念在生 命意义感和主观幸福感起部分中介作用。采用 Hayes 编写的 PROCESS3. 3 宏程序对正念中介作用的大小进 行检验, 设置 bootstrap $=5000$, 结果显示, 中介作用 的大小为 $0.03,95 \%$ 置信区间为 $[0.03,0.065]$, 置信 区间中不包括零，说明中介效应显著。

表 2 正念在生命意义感和主观幸福感间的中介作用检验

\begin{tabular}{|c|c|c|c|c|c|}
\hline & \multicolumn{2}{|c|}{ 中介变量（正念） } & \multicolumn{3}{|c|}{ 结果变量（主观幸福感） } \\
\hline 控制变量 & $\mathrm{M}_{2}$ & $\mathrm{M}_{3}$ & $\mathrm{M}_{0}$ & $\mathrm{M}_{1}$ & $\mathrm{M}_{4}$ \\
\hline 性别 & 0.05 & 0.04 & $-0.11^{*}$ & $-0.12^{*}$ & $-0.13^{*}$ \\
\hline 年龄 & 0.04 & 0.04 & -0.02 & -0.01 & -0.02 \\
\hline 年级 & -0.11 & -0.08 & $-0.24^{* * *}$ & -0.19 & $-0.18^{* *}$ \\
\hline 政治面貌 & $-0.16^{*}$ & $-0.15^{* *}$ & 0.01 & 0.03 & 0.06 \\
\hline 入学前身份 & $\begin{array}{l}-0.11 \\
\end{array}$ & -0.11 & 0.01 & 0.01 & 0.03 \\
\hline 自变量 & & & & & \\
\hline 生命意义感 & & $0.14^{* *}$ & & $0.22^{* * *}$ & $0.19^{* * *}$ \\
\hline 正念 & & & & & $0.20^{* * *}$ \\
\hline Adjust $\mathrm{R}^{2}$ & 0.04 & 0.05 & 0.05 & 0.10 & 0.13 \\
\hline $\mathrm{F}$ & $3.74^{* * *}$ & $4.42^{* * * *}$ & $5.07^{* * *}$ & $7.66^{* * *}$ & $8.90^{* * *}$ \\
\hline
\end{tabular}

\section{4. 讨论}

注: ${ }^{*}$ 表示 $p<0.05,{ }^{* *}$ 表示 $p<0.01,{ }^{* * *}$ 表示 $p<0.001$.

\section{1. 军校大学生生命意义感对主观幸福感的 正向影响}

研究发现, 军校学员生命意义感对主观幸福感具 有显著的促进作用, 也即军校学员感知到的生命意义 感越强, 主观幸福感也越强, 该结论在以往研究中也 有体现 ${ }^{[19]}$ 。意义管理理论也认为, 人类的存在需要生 活意义、人生目的和对世界的理解, 以及从复杂的人 生经历和经验中发掘或创造意义, 因此, 寻找和赋予 事物以意义是人类行为的重要动机。对于生命意义感 较强的军校大学生来说, 他们善于创造和发现意义, 能够用这些意义说服自己, 并将这些意义赋予具体的 事物和情境中, 即使在从事繁重工作、训练或生活不 如意时, 也能够从中找寻到意义, 从意义中汲取动力, 负重前行, 体会到更高的主观幸福感。此外, 有研究 表明, 生命意义感强的个体, 在追寻意义的过程中, 也往往能够建立起更加完善的人际关系, 在与人交往 过程中, 更能体会到来自他人的关心和帮助 ${ }^{[20]}$, 构建 强有力的社会支持网络, 进而促进其主观幸福感的提 高。

\section{2. 正念在军校大学生生命意义感和主观幸 福感间的中介作用}

研究结果进一步显示, 生命意义感除了能够直接 影响军校大学生的主观幸福感外, 还能通过正念的中 介作用间接影响主观幸福感, 也即正念在该过程中起 “桥梁” 作用, 部分传递了生命意义感对主观幸福感 的影响, 该结论对于进一步揭示生命意义感影响主观 幸福感的内部机制具有重要的理论价值。生命意义感 对正念的影响可从以下两个方面理解: 首先, 生命意 义感是一种能够给予个体方向感和价值感的目标, 与 目标感高度相关, 个体往往会将对生命意义的认知赋 予到具体的目标追寻过程中, 而目标的聚焦往往会促 使正念觉知水平的提升; 其次, 生命意义感较高的个 体并不会放弃对意义的寻受，而是会把寻找生命意义 作为对当下已获取意义感的完善和扩大, 进而获取更 深刻的意义理解 ${ }^{[21]}$, 而意义寻受的过程恰恰需要更高 的正念水平，从而发现或者创造新的意义。正念对主 观幸福感的影响比较容易理解, 正念水平较高的个体, 更容易接纳自身及周围环境, 能够平和地对待人生中 的挫折, 面对眼前困难时能够心无旁慗地应对, 即使 挫折暂时无法解决, 也能乐观看待, 对学习和工作保 持较高的满意度 ${ }^{[11]}$, 体验到更多的幸福感。此外, 正 念疗法认为心理问题产生的根源在于个体对待不同 
事物时产生的差别心，一旦产生了某个事物好或某个 事物不好的主观态度, 就容易引起心理问题, 而正念 疗法的作用原理就是要消除差别心，对出现的负性情 绪体验、观察、不评价，进而消除或缓解出现的心理 问题。毫无疑问，个体维持较高的正念水平对于克服 心理问题、维系心理健康具有重要的影响, 对提高和 维持较高的主观幸福感也具有重要的保护性作用。

\section{3. 对教育管理启示}

研究结论对军校大学生的教育管理也具有一定 的启示意义。与地方大学生相比, 军校大学生的身份 特点赋予其双重属性, 既要作为大学生完成必要的学 业, 又要履行军人使命, 聚焦备战打仗, 双重压力下 势必面临更高的心理危机风险。本研究通过问卷调查 及实证分析, 初步探索了影响军校大学生主观幸福感 的保护性因素, 也即生命意义感和正念在提升主观幸 福感方面具有显著的促进作用。鉴于此结论, 我们在 对军校大学生进行教育管理时, 可重点从以下几个方 面努力: 首先, 要注重提高军校大学生的生命意义感, 军校大学生首先是一名军人, 具有特殊的使命, 要注 重引导大学生从军人职能使命和个人实际相结合的 角度赋予当下学习、训练以特殊意义, 并从中获取不 竭的前进动力; 其次, 鉴于正念在该过程中的作用, 也可通过心理训练等形式, 也可立足实际, 教会学生 掌握诸如身体扫描、三分钟呼吸空间、静坐、正念饮 食、正念生活等正念训练方法, 以提高军校大学生的 主观幸福感。

\section{5. 结论}

本研究通过问卷调查及实证分析得出以下结论, 军校大学生生命意义感对主观幸福感具有显著的正 向促进作用, 正念在过程中起部分中介作用。对军校 大学生的教育管理及心理干预可重点从提高其生命 意义感和正念觉知水平入手, 合力发挥作用, 双管齐 下，共同促进其主观幸福感的提升。

\section{REFERENCES}

[1] Li Xin, Xu Jian. The management of military cadets should be paid more attention to the cultivation of psychological protection ability in the new era [J]. Political work journal, 2019(9):49-51.

[2] Diener, Ed. Subjective well-being $[\mathrm{J}]$. Psychological Bulletin, 1984,95(3):542-575.

[3] Diener E, Ryan K. Subjective Well-Being: A General Overview[J]. South African Journal of Psychology, 2009,39(4):391-406.

[4] George L S, Park C L. Meaning in Life as Comprehension, Purpose, and Mattering: Toward Integration and New Research Questions[J].
Review of General Psychology, 2016,20(3):205220.

[5] Heintzelman S J, King L A. Routines and Meaning in Life[J]. Personality and Social Psychology Bulletin, 2018,45:688-699.

[6] Niu duan, Huang wei. The Effects of Meaning in Life on Mindfulness and Subjective Happiness of Science-Technology Talents[J]. Journal of southwest jiaotong university (Social Sciences), 2019,20(4):1-10.

[7] McKnight, Kashdan P E, Todd B. Purpose in life as a system that creates and sustains health and well-being: An integrative, testable theory[J]. Review of General Psychology, 2009,13(3):242251.

[8] Chiodelli R, Mello L T N, Jesus S N, et al. Effects of a brief mindfulness-based intervention on emotional regulation and levels of mindfulness in senior students[J]. Psicologia Reflexo E Crítica, 2018,31(1):1-10.

[9] Dane, E. Paying Attention to Mindfulness and Its Effects on Task Performance in the Workplace[J]. Journal of Management, 2011,37(4):997-1018.

[10] Liu Siman, Liu Keting, Li Tiantian.etc. The Impact of Mindfulness on Subjective Well-being

of College Students: The Mediating Effects of Emotion Regulation and Resilience[J]. Journal of psychological science, 2015,38(4):889-895.

[11] Wang Qinfei. The relation and intervention among mindfulness, psychological capital and meaning in life of undergraduates[D]. HeNan University, 2019.

[12] Huang Ling. Correlation of mindfulness perception stress and sense of meaning of life in postoperative patients with colorectal cancer[D]. YanBian University, 2019.

[13] Hutzell R R. The Continued Relevance of Man's Search for Meaning[J]. PsycCRITIQUES, 2006,51(5):102-112.

[14] Steger M F, Frazier P, Oishi S, et al. The meaning in life questionnaire: Assessing the presence of and search for meaning in life $[\mathrm{J}]$. Journal of Counseling Psychology, 2006,53(1):80-93.

[15] LIU Sisi, GAN Yiqun. Reliability and validity of the Chinese version of the Meaning in Life Questionnaire[J]. Chinese Mental Health Journal, 2010,24(6):478-482.

[16] Brown K W, Ryan R M. The benefits of being present: mindfulness and its role in psychological 
well-being[J]. Journal of Personality and Social Psychology, 2003,84(4):822-848.

[17] Wang Xiangdong, Wang Xilin, Ma Hong. Manual of mental health rating scales[M]. Chinese Mental Health Journal, 1999.

[18] Wen Zhonglin, Zhang Lei, Hou Jietai, etc. Testing and application of the mediating effects[J]. Acta Psychological Sinica, 2004(5):614-620.

[19] Shen Linlin, Zhang Zhen. Relationship between Grandparenting Willingness and Grandparents'Subjective Well-being-Mediating Role of Family Cohesion and Meaning in Life[J]. Chinese Journal of Clinical Psychology, 2020,28(04):834-839.

[20] Li Zining. The Influence of the Sense of Life Meaning on the Attitude to Death in the Elderly: The Mediating Role of Social Support[D]. JiLin University, 2019.

[21] Xiong Pengdi, Yang Le, Zhu Zhuohong. Relation of posttraumatic growth to meaning of life and

experiential avoidance in adolescents[J]. Chinese Mental Health Journal, 2015,29(1):40-46. 